\title{
Eficacia del contacto e información como técnicas de cambio de actitudes hacia personas con discapacidad en niños de Educación Primaria*
}

\section{Effectiveness of Contact and Information as Attitudes Change Techniques towards People with Disabilities in Primary School Children}

Recibido: enero 11 de 2012 | Revisado: junio 28 de 2012 | Aceptado: agosto 15 de 2012

\author{
María Ángeles Alcedo RodríGueZ** \\ LAURA ElísABet GÓMEZ SÁNCHEZ *** \\ ANTONIO LEÓn AgUAdo DÍAZ ***** \\ Universidad de Oviedo, España \\ BENITO ARIAS MARTÍNEZ***** \\ Universidad de Valladolid, España \\ Rosalía GONZÁlez GARCÍA ******* \\ Colegio Vega de Guceo (Turón), España
}

\section{doi:10.11144/Javeriana.UPSY12-2.ecit}

Para citar este artículo: Alcedo, M. A., Gómez, L. E., Aguado, A. L., Arias, B. \& González, R. (2013). Eficacia del contacto e información como técnicas de cambio de actitudes hacia personas con discapacidad en niños de Educación Primaria. Universitas Psychologica, 12(2), 493-504.

Artículo de investigación realizado con el apoyo de la Universidad de Oviedo, España.

** Universidad de Oviedo, España. Doctora en Psicología y Profesora Titular del Departamento de Psicología. E-mail: malcedo@uniovi.es

**** Universidad de Oviedo, España. Doctora en Psicología y Profesora Ayudante, Doctora del Departamento de Psicología. E-mail: gomezlaura@ uniovi.es

***** Universidad de Oviedo, España. Doctor en Psicología y Profesor Titular del Departamento de Psicología E-mail: aaguado@uniovi.es

******* Universidad de Valladolid, España. Doctor en Psicología y Profesor Titular del Departamento de Psicología.E-mail: barias@psi.uva.es

******** Colegio Vega de Guceo (Turón), España. Doctora en Psicología por la Universidad de Oviedo. Directora, España.E-mail: rosaliaglez@hotmail. com

\section{R E S U M E N}

La actitudes negativas hacia las personas con discapacidad imperantes en la sociedad plantean la necesidad de intervenciones que modifiquen tales actitudes. Con este objetivo se ha desarrollado un programa de cambio de actitudes hacia personas con discapacidad en alumnos de educación primaria, 100 niños y niñas, entre 7 y 10 años, asignados 39 al grupo experimental y 61 al grupo control. Se utilizaron como técnicas de cambio la información y el contacto. También se efectuó un seguimiento de dos años y medio, durante el que se realizaron actividades recordatorio. El instrumento de evaluación utilizado fue la Escala de Actitudes hacia Personas con Discapacidad (Verdugo, Arias \& Jenaro, 1994). Los resultados alcanzados abogan por la eficacia de estas técnicas, al encontrase entre las medidas pretratamiento y postratamiento diferencias estadísticamente significativas para estas últimas a favor del grupo experimental. Tales cambios se mantienen en el seguimiento.

Palabras clave autores

Actitudes, discapacidad, cambio de actitudes, evaluación, intervención.

Palabras clave descriptores

Psicología educativa, investigación cuantitativa, diseño cuasi-experimental.

\section{A B S T R A C T}

The negative attitudes toward people with disabilities prevailing in our society suggest the need for interventions to modify those attitudes. With this aim a program in changing attitudes towards people with disabilities in primary school children has been developed. 100 children between 7 and 10 years old took part in the study, 39 were assigned to the experimental group and 61 to the control group. Information and contact have been used as change techniques. It has also been followed up for two years and a half. During this time, activities reminders have been made. The assessment instrument used was the Scale of Attitudes toward People with Disabilities (Verdugo, Arias \& Jenaro, 1994). The results obtained argue for the effectiveness of these techniques. There are statistically significant differences between pretreatment and posttreatment measures in favor of 
the experimental group. Also, these changes at follow up have been maintained.

Key words authors

Attitudes, disability, change of attitudes, assessment, intervention. Key words plus

Educative Psychology, Quantitative Research, Quasi-experimental Design.

\section{Introducción}

El primer Informe Mundial sobre la Discapacidad, publicado recientemente por la Organización Mundial de la Salud (OMS) junto al Grupo del Banco Mundial (2011), y elaborado con el propósito de proporcionar una base empírica sobre la que asentar y formular políticas y programas innovadores que mejoren las vidas de las personas con discapacidad, destaca que entre los principales obstáculos para garantizar la inclusión, la autonomía y los derechos de estas personas se encuentran las actitudes negativas. Las creencias y prejuicios constituyen barreras para la educación, el empleo, la atención de salud y la participación social. La necesidad de modificar dichas actitudes y la de favorecer y promover activamente la no discriminación viene siendo reconocida y demandada no solo en los estudios y trabajos específicos centrados en esta temática (Flórez, Aguado \& Alcedo, 2009; Nowicki, 2006), sino también desde estamentos educativos y organismos e instituciones variados que potencian la promulgación y el posterior desarrollo normativo de leyes que abordan específicamente este tema (Estatuto del Estudiante Universitario, 2010; Boletín Oficial del Estado, 2007). El citado Informe Mundial sobre la Discapacidad plantea, entre sus recomendaciones para favorecer una sociedad inclusiva, fomentar la sensibilización pública y la comprensión de la discapacidad a través de la puesta en marcha de campañas que ayuden a cambiar las actitudes frente a las cuestiones estigmatizadas tan presentes aún en la sociedad (OMS \& Banco Mundial, 2011).

Así pues, la persistencia de prejuicios y estereotipos hacia las personas con discapacidad, que no favorecen el respeto y la comprensión mutua y potencian el rechazo y la marginación, consta- tan la relevancia social y aplicada de este tema y justifica la necesidad de un cambio y la puesta en marcha de intervenciones encaminadas a dicho objetivo. Sin embargo, y pese a que se dispone de apoyo empírico acerca de la posibilidad de modificar y mejorar dichas actitudes (Flórez et al., 2009; Hodge, Davis, Woodard \& Sherrill, 2002; Krahé \& Altwasser, 2006; McCarthy \& Light, 2005; Morton \& Campbell, 2007; Slininger, Sherrill \& Jankowski, 2000), los estudios centrados en la implementación de estos programas y en la valoración de su eficacia siguen siendo una estrategia de intervención no muy difundida. Y ello, pese a haberse constatado que estas actitudes negativas aparecen a edades muy tempranas (Magiati, Dockrell \& Longotheti, 2002; Maras \& Brown, 2000; Nowicki \& Sandieson, 2002), lo que enfatiza la necesidad de prevención. Asimismo, esta formación temprana también apoya la implementación de programas de cambio de actitudes a edades en las que habitualmente no se suele trabajar, es decir, en los primeros años de escolarización de estos niños.

Otra de las razones decisivas que justifican la realización de este tipo de programas estriba en que un aspecto básico y elemental de este proceso de integración, como es la inclusión escolar, no cuente con actitudes muy favorables. Los estudios señalan que coexisten actitudes de aceptación y de rechazo, y que sería necesario que los centros escolares tuviesen una estructura que promoviese el éxito en las tareas y las relaciones positivas entre estudiantes con y sin discapacidad (McDougall, De Wit, King, Miller \& Killip, 2004). También se hacen necesarias intervenciones que proporcionen la información necesaria y destruyan los estereotipos erróneos que dificultan la integración educativa de los niños con discapacidad. La tolerancia, respeto y aceptación de la persona con discapacidad es mayor en la medida en que existen más oportunidades de conocer y comprender lo que significa la discapacidad; la empatía y el acercamiento afectivo propician actitudes más positivas (García \& Hernández, 2011; Laws \& Kelly, 2005; Polo, Fernández \& Díaz, 2011). Que los niños acudan a clase con sus compañeros con discapacidad, sin más, no facilita un cambio de actitudes (Navas, Torregrosa 
\& Mula, 2004; Nikolaraizi \& De Reybekiel, 2001; Wong, 2008). De hecho, una de las apuestas más firmes de la mayoría de los países occidentales ha sido y sigue siendo la inclusión educativa de los niños con discapacidad, pero los resultados de este proceso no están siendo todo lo positivos que en un principio se postulaban (Huete, Sola \& Lara, 2010; OMS \& Grupo del Banco Mundial, 2011; Peralta, 2007). Y parece que las actitudes no son ajenas a estos resultados. Se ha comentado y reiterado con frecuencia que una plena integración de los niños con discapacidad depende, en ocasiones, tanto o más de las actitudes de compañeros y profesores que del diseño de un currículum bien adaptado. Existe amplio consenso a la hora de admitir que el éxito de la escuela inclusiva está directamente relacionado con las actitudes positivas de la comunidad educativa (Rillota \& Nettelbeck, 2007).

Lo expuesto hasta este momento, constituye un conjunto de argumentos justificados para la puesta en marcha de trabajos como el que se presenta. Promover un cambio de actitudes hacia esta población no solo busca tener un efecto inmediato en aquellas aulas con alumnos que presenten alguna necesidad educativa, sino que también se debe valorar su efecto a más largo plazo, cuando los niños, ya adultos, contacten e interactúen con personas con discapacidad.

De acuerdo con lo anterior, la presente investigación responde a los siguientes objetivos: (a) comprobar si la aplicación de un programa estructurado, para niños de edades entre 7 y 10 años, resulta eficaz para la mejora de las actitudes hacia personas con discapacidad; (b) comprobar si los efectos de la intervención (i. e., actitudes positivas hacia la discapacidad) se mantienen en el tiempo (i. e., seguimiento a lo largo de dos años y medio); (c) analizar si las actitudes hacia la discapacidad se mantienen más positivas en función de si se realizan actividades de tipo recordatorio.

Para la comprobación de estos objetivos se parte de las siguientes hipótesis: H1: no existirán diferencias significativas previas al tratamiento entre el grupo experimental (GE) y el grupo control (GC); H2: los alumnos participantes en el programa de cambio de actitudes hacia personas con discapa- cidad presentarán una valoración más positiva de las actitudes, de forma que, entre la medida pretratamiento y la primera medida postratamiento, las puntuaciones serán favorables hacia esta última para el GE; H3: se espera mantenimiento temporal de dicha mejora; en consecuencia, la ganancia conseguida por el GE una vez aplicado el programa de tratamiento se mantendrá a lo largo del tiempo, es decir, en los sucesivos postest. Esta tendencia será más favorable al introducir actividades recordatorio.

\section{Metodología}

\section{Diseño}

En este estudio se ha utilizado un diseño cuasiexperimental de grupo control no equivalente con pretest y postest, que consta de un factor intrasujeto con seis niveles (i. e., momentos de evaluación) y un factor intersujeto (i. e., pertenencia al grupo experimental o al grupo control). Así, la variable independiente o tratamiento consiste en un programa de cambio de actitudes hacia las personas con discapacidad y la variable dependiente, en la evaluación de tales actitudes hacia la discapacidad mediante una prueba psicológica: la Escala de Actitudes hacia Personas con Discapacidad (EAPD) de Verdugo, Arias y Jenaro (1994), que se describirá más adelante.

\section{Participantes}

La muestra estuvo compuesta por alumnado $(N=100)$ que asistía a tercer y cuarto curso de Educación Primaria en tres colegios públicos ubicados en la zona centro del Principado de Asturias (España). Los tres centros están situados en localidades próximas, con características sociodemográficas, económicas y culturales muy similares, todas ellas pertenecientes a una de las cuencas mineras del centro de Asturias. El motor principal de su economía eran las minas de carbón hasta su cierre progresivo a partir de los años 90. Esto provocó una importante pérdida de población y un aumento en la edad media de sus habitantes. En el momento del inicio del estudio, año 2008, el porcentaje medio 
de población de estas localidades estaba en torno a los 3.500 habitantes. Los padres de estos alumnos comparten, asimismo, un nivel económico medio, con una renta per cápita muy similar, ya que en su mayoría son familias con ingresos de salarios de la minería, y un nivel socio cultural medio-bajo (Instituto Asturiano de Estadística, 2011). Por lo que se refiere a las características de los colegios participantes, los tres son centros con aulas poco numerosas (entre 15 y 20 alumnos por aula), en las que todos los alumnos siguen los mismos planes de estudios, y cuya tarea se haya supervisada por el mismo inspector de zona. Las características físicas de estos centros también son similares: edificios de unos 25 años de antigüedad, ubicados dentro del núcleo urbano de la localidad.

Dichos centros se ofrecieron a trabajar voluntariamente en el programa, por lo que se partió de grupos naturales dentro del entorno escolar (i. e., muestreo incidental). El $52 \%$ eran niñas y el $48 \%$ niños, cumpliéndose la hipótesis de equiprobabilidad $\left(\chi^{2}=0.16 ; p=0.689\right)$. El rango de edad osciló entre los 7 y los 10 años $(M=8.28 ; D E=0.726)$; la moda fue de 8 años en el caso de las niñas y de 9 años en el caso de los niños. En los tres centros educativos existía integración de alumnado con discapacidad, principalmente con discapacidad auditiva y visual.

El grupo experimental (GE) estaba formado por las 39 personas $(\mathrm{N}$ niños $=21 ; 53.8 \%$ ) $(\mathrm{N}$ niñas $=18 ; 46.2 \%)$ de uno de los centros escolares participantes. Entre ellos, el $28.2 \%$ manifestó tener conocimientos sobre discapacidad y el $41 \%$ declaró que conocía o se había relacionado con alguna persona con discapacidad. El grupo control (GC) quedó constituido por 61 sujetos $(\mathrm{N}$ niños $=27 ; 44.3 \%)(n$ niñas $=34 ; 55.7 \%)$ de los dos centros educativos restantes. El $47.5 \%$ de los escolares de este $\mathrm{GC}$ refirió no tener conocimientos sobre discapacidad, y el $67.2 \%$ que no conocía ni se había relacionado con personas con discapacidad.

\section{Instrumento}

El instrumento de evaluación utilizado fue, como se ha señalado, la Escala de Actitudes hacia
Personas con Discapacidad (EAPD) de Verdugo et al. (1994). Se trata de una escala compuesta por 31 afirmaciones sobre diferentes aspectos relacionados con las personas con discapacidad. El formato de respuesta es una escala Likert de tres opciones ('verdadero', 'falso', 'no sé'). Su objetivo consiste en evaluar las actitudes que el alumnado sin discapacidad mantiene hacia sus pares con discapacidad, de tal forma que posibilita ahondar en el entendimiento de la naturaleza de la interacción entre ambos grupos, tanto en sus aspectos negativos (p. ej., rechazo, evitación, marginación, etc.) como positivos (p. ej., amistad, cooperación, tolerancia).

Aunque los autores de la escala han proporcionado en el estudio de validación suficientes evidencias acerca de los criterios de bondad de la EAPD para evaluar las actitudes (Verdugo et al., 1994), bien es sabido que las propiedades psicométricas de un instrumento varían en función de la muestra a la que se aplica. Por esto, se quiso comprobar la adecuación de la escala en los participantes de este estudio. Se procedió, por tanto, al cálculo del coeficiente alfa de Cronbach en las seis aplicaciones del instrumento. Los valores obtenidos oscilaron entre 0.767 y 0.864 , sin existir diferencias significativas entre ellos $\left(\chi_{(5)}^{2}=8.144 ; p>0.05\right)$, por lo que se pudo concluir que la consistencia interna de la EAPD en esta muestra es adecuada.

\section{Procedimiento}

La cronología del programa de intervención se resume en el Tabla 1. La implementación del mismo se ha desarrollado a lo largo de un trimestre escolar, mediante nueve sesiones semanales de hora y media cada una en el GE. El programa fue aplicado por un psicólogo especialista en clínica, con el apoyo de dos estudiantes de psicología, que previamente habían sido entrenados. Todas las sesiones se realizaron en horario escolar, el mismo día de la semana y a la misma hora. Este programa formaba parte de las actividades complementarias del currículo académico del Centro. La primera sesión de intervención coincidió con la primera evaluación de las actitudes en el GE. 
Una vez finalizado el programa se llevó a cabo la primera evaluación postest.

Dado que en estudios previos los resultados indicaban que la ganancia en la mejora de las actitudes conseguida disminuía con el paso del tiempo (Aguado, Alcedo \& Flórez, 2003, 2004; Rillota \& Nettelbeck, 2007; Shevlin \& O’Moore, 2000), especialmente tras un año desde la finalización del programa, se llevó a cabo una actividad recordatoria por trimestre (i.e., un total de siete actividades) durante los dos años y medio que duró el período de seguimiento.

En cuanto a las evaluaciones postest, la primera medida postratamiento se realizó una semana después de la finalización del programa; la segunda y tercera, tres y seis meses después, respectivamente. La cuarta y quinta medidas postest tuvieron lugar a los 21 y 30 meses del primer pretest.

Los contenidos de las distintas sesiones y actividades recordatorio se centraron en los siguientes objetivos: (a) conocer las diferentes discapacidades, no desde una perspectiva negativa centrada en la pena y lástima, sino mostrando el lado positivo de ajuste y posibilidades de las personas con discapacidad; (b) permitir que el alumnado comprobara por sí mismo la información que estaba recibiendo en el programa mediante el contacto directo con personas con discapacidad; (c) sensibilizar al alumnado acerca de las limitaciones y trabas que suponen las barreras arquitectónicas y actitudinales en el proceso de integración de las personas con discapacidad; (d) enseñar al alumnado a interactuar de forma adecuada con personas con discapacidad, de forma que puedan producirse intercambios enriquecedores y agradables para todos.

La estructura de las distintas sesiones era muy similar en cuanto a su formato. Se iniciaba la sesión con la presentación del tema a debatir, generalmente aportando información específica y concreta sobre el mismo. La técnica de "lluvia de ideas" permitía que los alumnos expusieran sus conocimientos, ideas, e incluso emociones y sentimientos hacia el tema, a la vez que potenciaba que todos los niños participasen con algún tipo de aportación personal. Estas intervenciones permitían al terapeuta introducir y/o reconducir la información recogida hacia los contenidos y objetivos propuestos. El desarrollo de las sesiones proseguía con la introducción de materiales (vídeos, cuentos, ayudas técnicas) y/o actividades (charlas-coloquio con personas con discapacidad, visitas a centros) que potenciaban el contacto directo o indirecto con el ámbito de la discapacidad, siempre desde una perspectiva de apoyo a la inclusión y normalización. Cada sesión finalizaba con una discusión guiada a modo de resumen sobre el tema concreto que se había experimentado y aprendido. Frecuentemente, los niños realizaban dibujos relacionados con la temática abordada que quedaban expuestos en un panel acondicionado específicamente para el programa.

Las técnicas de cambio de actitudes utilizadas para la consecución de estos objetivos fueron la información directa e indirecta sobre las personas con discapacidad y el contacto directo con personas con distintos tipos de discapacidad. Se eligieron este tipo de estrategias dada su eficacia en estudio previos (Aguado et al., 2003, 2004; Almazán, 2003; Hodge et al., 2002; McCarthy \& Light, 2005; Morton \& Campbell, 2007; Slininger et al., 2000; Rillota \& Nettelbeck, 2007).

\section{Análisis de datos}

Los datos fueron analizados con el programa estadístico SPSS versión 19.0. En primer lugar se analizó la fiabilidad del instrumento de evaluación (EAPD) en la muestra de estudio en términos de consistencia interna (mediante el coeficiente alfa de Cronbach). Una vez comprobada la fiabilidad, se procedió a la comprobación de las hipótesis mediante la prueba $t$ de Student para grupos independientes y mediante el modelo mixto de medidas repetidas.

\section{Resultados}

En primer lugar se analizó la situación de partida de los niños del GE y del GC, con el fin de comprobar la hipótesis de que no existían diferencias significativas en las actitudes hacia las personas con discapacidad entre ambos grupos previas al tratamiento (i. e., evaluación pretest). Contraria- 
mente a lo esperado, la media del GC fue superior a la del GE (Tabla 2), lo que indicaba la presencia de actitudes más positivas en el primer grupo. No obstante, tales diferencias no resultaron significativas $(t=1.923 ; p>0.05)$, por lo que se confirma la primera hipótesis.

Una vez comprobado este primer requisito de equivalencia entre los grupos, se sometió a prueba la hipótesis relativa a que los participantes en el programa (GE) mostrarían actitudes significativamente más positivas en la primera medida postest que en la medida pretest. En este caso, la prueba de comparación de medias resultó significativa $(t=-3.115$; $p<0.05)$ y a favor de esta segunda hipótesis. Este resultado estaría acorde con la hipótesis de eficacia del programa de intervención. La tercera hipótesis planteaba el mantenimiento temporal de la mejora de las actitudes tras el programa. Se esperaba, por tanto, que la ganancia conseguida por el GE se mantuviera a lo largo del tiempo, una vez finalizado el tratamiento, en los sucesivos postest. Asimismo, se presuponía que las puntuaciones en actitudes en el GE siguieran siendo significativamente superiores a las obtenidas por el GC. Para su comprobación se realizó un modelo de medidas repetidas mixto, con cinco postest de seguimiento, que dio lugar a diferencias significativas tanto en las puntuaciones de la escala en los distintos momentos $\left(F_{5,68}=10.249\right.$; $p<0.05)$ como en esta tendencia en función de la presencia o ausencia del tratamiento $\left(F_{5,68}=3.442\right.$, $p<0.05)$. Los contrastes polinómicos mostraron una tendencia lineal en las puntuaciones de ambos grupos, con diferencias significativas entre las ganancias conseguidas por el GE y el GC a favor del primero $\left(F_{1,72}=10.681 ; p<0.05\right)$.

$\mathrm{Al}$ analizar las puntuaciones de los grupos en cada momento del seguimiento, como se muestra en la Figura 1, se aprecia que el GE, aun partiendo de puntuaciones inferiores pero no significativas en el pretest, obtiene en todos los postest puntuaciones

TABLA 1

Cronología y secuencia de la investigación

\begin{tabular}{llc}
\hline \multicolumn{1}{c}{ Tipo } & \multicolumn{1}{c}{ Contenido } & Grupo \\
\hline Sesión 1 & Presentación y Pretest (IAPD y EAPD) & GE y GC \\
Sesión 2 & Conferencia de Experto & GE \\
Sesión 3 & Visionado de videos sobre discapacidad & GE \\
Sesión 4 & Visita a un Colegio de Educación Especial & GE \\
Sesión 5 & Visita a un Centro Ocupacional de Empleo & GE \\
Sesión 6 & Charla-coloquio con personas con discapacidad física & GE \\
Sesión 7 & Charla-coloquio con personas con discapacidad visual & GE \\
Sesión 8 & Charla-coloquio con personas con discapacidad auditiva & GE \\
Sesión 9 & Visita a un Centro Especial de Empleo & GE \\
Postest 1 & Primera evaluación postratamiento & GE y GC \\
Recordatorio 1 & Realización de un artículo sobre el programa & GE \\
Postest 2 & Segunda evaluación postratamiento & GE y GC \\
Recordatorio 2 & Coloquio sobre discapacidad física & GE \\
Postest 3 & Tercera evaluación postratamiento & GE y GC \\
Recordatorio 3 & Coloquio sobre discapacidad auditiva & GE \\
Recordatorio 4 & Juegos con personas con discapacidad física & GE \\
Recordatorio 5 & Video sobre diferentes discapacidades & GE \\
Postest 4 & Cuarta evaluación postratamiento & GE y GC \\
Recordatorio 6 & Coloquio sobre discapacidad visual & GE \\
Recordatorio 7 & Obra de teatro sobre discapacidad & GE \\
Postest 5 & Quinta evaluación postratamiento & GE y GC \\
\hline
\end{tabular}

Fuente: elaboración propia. 


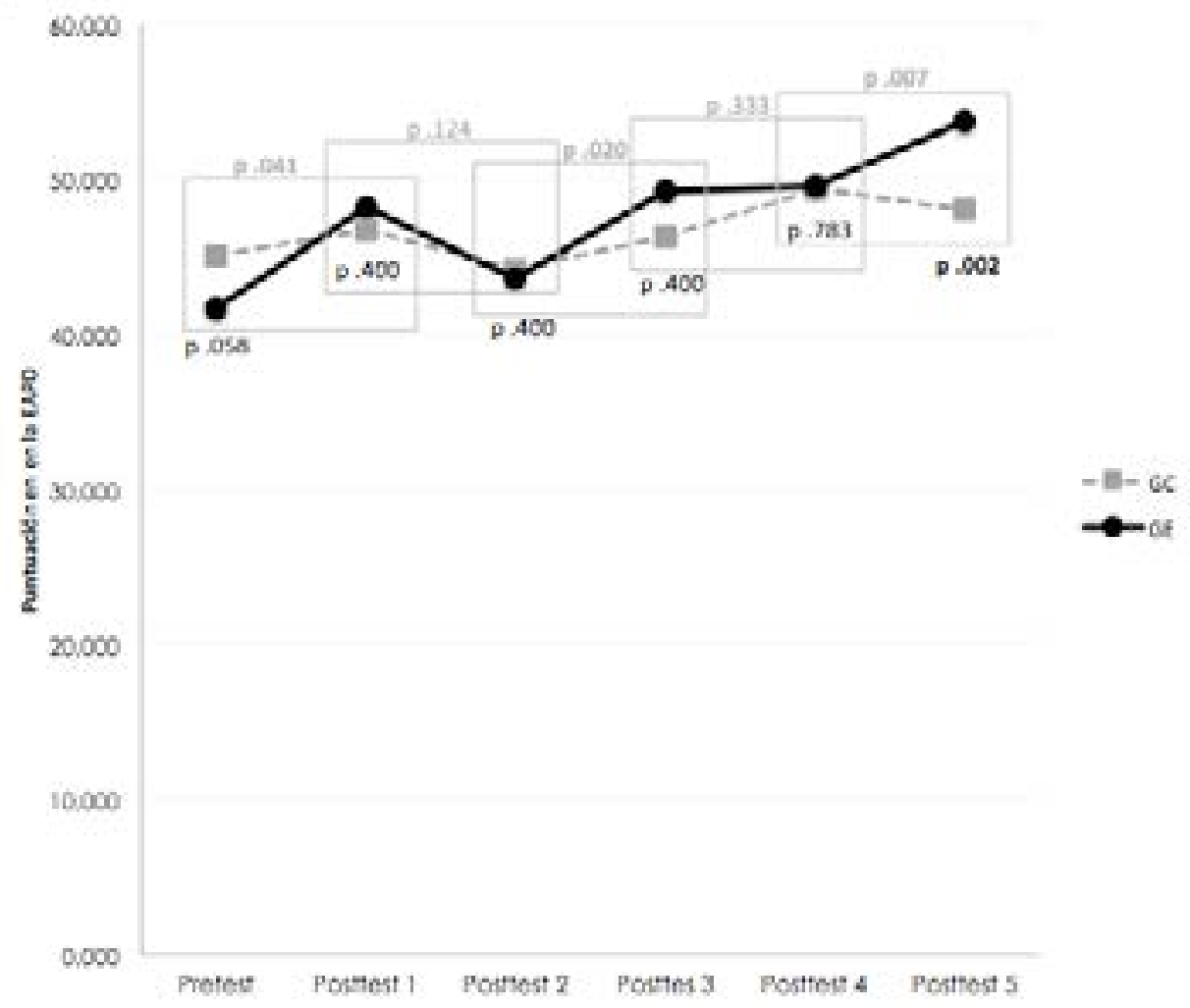

Figura 1. Comparación de medias entre GC y GE en los seis momentos y entre los momentos de evaluación.

Nota. Las probabilidades asociadas de los contrastes entre las medias obtenidas por el GE y el GC en cada uno de los seis momentos se han coloreado en negro. Las probabilidades asociadas de los contrastes entre las medias de los grupos entre dos momentos de evaluación (cuyas medias se representan mediante dos marcadores contenidos en un mismo recuadro) se han coloreado en gris. Las probabilidades asociadas que resultaron significativas a un nivel de confianza del $95 \%$ se han destacado en negrita en ambos casos.

Fuente: elaboración propia.

superiores al GC (con la excepción del segundo postest), aunque estas diferencias únicamente alcanzan significatividad en el último postest $(p<$ 0.05). Si se relaciona esta evolución de las puntuaciones con el tratamiento, se observa que existen diferencias significativas entre el pretest y el postest

\section{TABLA 2}

Medias en el pretest y primer postest

\begin{tabular}{lccc}
\hline & M & DE & $\begin{array}{c}\text { Error típ. } \\
\text { de media }\end{array}$ \\
\hline G.E. & & & \\
$\quad$ Pretest & 41.600 & 8.246 & 1.393 \\
$\quad$ Postest1 & 48.216 & 9.294 & 1.528 \\
G.C. & & & \\
$\quad$ Pretest & 45.120 & 8.348 & 1.180 \\
$\quad$ Postest 1 & 46.642 & 8.426 & 1.125 \\
\hline
\end{tabular}

Fuente: elaboración propia.
$1(F=4.332, p<0.05)$. Sin embargo, entre el postest 1 y el 2 estas diferencias no son significativas $(F=2.417 ; p>0.05)$. De hecho, en este momento de evaluación las puntuaciones empeoran en ambos grupos. Entre el postest 2 y el 3 se recupera la significatividad de las puntuaciones $(F=5.647$; $p<0.05)$, perdiéndose de nuevo entre el postest 3 y el $4(F=0.948, p<0.05)$, para volver a recuperarse en la última medida $(F=7.708 ; p<0.05)$.

Se puede concluir, por tanto, que existe relación entre el tratamiento y el mantenimiento de actitudes positivas hacia la discapacidad a lo largo del tiempo, lo que puede considerarse como una evidencia a favor de la eficacia del programa. Aunque en algunos momentos se ha perdido significación estadística, esta se mantiene al final del tratamiento, incluso con una diferencia de tendencia en las 
puntuaciones de los grupos favorable al GE en la última evaluación.

Por último, se comprobó la eficacia de las actividades recordatorio realizadas a lo largo del seguimiento. Para esto, se realizó una prueba de contraste de medias entre el pretest y el primer postest, $y$ entre este y el último postest. En este caso, como se observa en la Tabla 3, tanto el GE experimental como era esperado $\left(t_{(32)}=-3.115 ; p=0.004\right)-$ como el GC -en contra de lo esperado $\left(t_{(46)}=-2.130\right.$; $p=0.039)$ - mejoraron significativamente sus puntuaciones entre el pretest y el primer postest, a un nivel de confianza del $95 \%$. Sin embargo, en el último postest las puntuaciones obtenidas por ambos grupos se alejaron sensiblemente, con una mejora de las actitudes significativamente superior en el $\mathrm{GE}\left(t_{(33)}=-2.130 ; p=0.039\right)$, mientras las actitudes empeoraban, aunque no de forma significativa, en el GC $\left(t_{(54)}=-3.115 ; p=0.241\right)$. El hecho de que las puntuaciones mejoren progresivamente en el GE, hasta llegar a ser significativas las diferencias en comparación con el primer y el último postest, puede considerarse un dato a favor de la eficacia de las actividades recordatorio realizadas en el GE a lo largo de los dos años y medio que duró el período de seguimiento.

\section{Discusión y conclusiones}

El primer objetivo del trabajo se centró en comprobar la equivalencia inicial de los grupos, verificándose esta al no aparecer diferencias significativas previas a la implementación del programa entre el GE y GC. Así pues, el alumnado participante en el programa, a pesar de pertenecer a centros escolares diferentes, muestran una equiparación inicial en lo que a sus actitudes hacia las personas con discapacidad se refiere. El siguiente objetivo consistió en determinar el grado de efectividad del programa de cambio de actitudes. Los resultados univariados aportados por la prueba $t$ de muestras independientes señalaban que tanto el GE como el GC experimentan una mejora significativa de las actitudes entre la medida pretratamiento y la primera medida postratamiento, aunque esta es mayor en el GE. Esta circunstancia obliga a considerar la presencia de variables extrañas que, además del programa de cambio de actitudes, estén influyendo de algún modo en los resultados obtenidos. De todas estas, la explicación más plausible pudiera encontrarse en el contacto de los alumnos del GC con sus pares con discapacidad, mientras que el GE participaba en el programa de cambio de actitudes. No se debe olvidar que al ser compañeros, en muchos casos no solo de centro educativo sino también de aula, los participantes del GC compartían actividades conjuntas con sus compañeros con discapacidad, tanto las organizadas en horario escolar como extraescolar. La influencia de esta variable no ha podido ser analizada al ser una constante (i. e, tanto en el GC como en GE ha existido contacto del alumnado con personas con discapacidad), pero sus efectos podrían explicar, al menos en parte, los resultados alcanzados. No obstante, el GC, que ha mantenido contacto permanente y que ya partía de puntuaciones superiores en el pretest, presenta en casi todas las medidas postests, a excepción del segundo postest, puntuaciones inferiores a las del GE. Estos datos apoyarían los resultados encontrados en estudios previos acerca de que el contacto estructurado y la

TABLA 3

Diferencias de medias relacionadas: pretest, postest 1 y postest 5

\begin{tabular}{lcccccc}
\hline & M & DE & Error típ. de la media & $t$ & gl & Sig. (bilateral) \\
\hline G.E. & & & & & & \\
Pre y post 1 & -5.515 & 5.108 & 1.770 & -3.115 & 32 & 0.004 \\
Post 1 y post 5 & -5.764 & 6.214 & 1.066 & -5.410 & 33 & 0.000 \\
G.C. & & & & & & \\
Pre y post 1 & -2.127 & 6.848 & 0.999 & -2.130 & 46 & 0.039 \\
Post 1 y post 5 & -1.745 & 10.910 & 1.471 & -1.186 & 54 & 0.241 \\
\hline
\end{tabular}

Fuente: elaboración propia. 
realización de actividades que aportan información sobre la discapacidad son estrategias más eficaces que el mero contacto o convivencia (Aguado et al., 2003, 2004; Aguado, Alcedo \& Arias, 2008; Fisher-Polites, 2004; Hodge et al., 2002; Krahé \& Altwasser, 2006; Maras \& Brown, 2000; McCarthy \& Light, 2005; Shevlin \& O’Moore, 2000; Slininger et al., 2000; Wong, 2008).

El contacto estructurado y la información permiten acercarse a la realidad de la discapacidad de una manera más adecuada, con expectativas más ajustadas, y contribuyen a eliminar barreras y prejuicios. Sin embargo, a pesar de que este contacto estructurado no se produjo en el GC, se considera que la hipótesis de contacto permite a su vez explicar la mejora de las actitudes entre el pretest y el primer postest. Es decir, aunque el contacto no ha sido estructurado puede haber sido lo suficientemente intenso y satisfactorio, de forma que ha propiciado actitudes más positivas. Similares planteamientos también aparecen recogidos en la bibliografía especializada (Diamond, Hestenes, Carpenter \& Innes, 1997; Manetti, Schenider \& Siperstein, 2001; Pettigrew \& Trop, 2006).

Otro de los objetivos de este trabajo consistía en la comprobación del mantenimiento temporal de estos cambios de actitudes. Dado que los resultados aportados por estudios similares que han realizado seguimiento muestran que la efectividad del programa va perdiendo significación estadística con el paso del tiempo, especialmente tras haberse cumplido un año de la finalización del programa (Aguado et al., 2003, 2004; Rillota \& Nettelbeck, 2007; Shevlin $\&$ O'Moore, 2000), se introdujeron varias sesiones recordatorio para favorecer el recuerdo y comprobar si existía mejora en los resultados del programa a más largo plazo. Los datos de la EAPD mostraron diferencias significativas a favor del GE a lo largo del seguimiento en el primer, tercer y último postest. Es más, entre el postest 4 y el 5 las puntuaciones del GE seguían mejorando mientras que las del GC empeoraron. Es decir, en los niños del GE las actitudes positivas no solo perduran, sino que incluso mejoran con el paso del tiempo, una consecuencia que se puede atribuir a una mayor sensibilidad tras participar en un programa de cambio de actitudes.
Resultados similares se recogen en el programa de Aguado et al. (2008), que trabaja con niños de edades similares.

Respecto al descenso en las puntuaciones del GC, una posible explicación radica en el planteamiento defendido por algunos autores (Burns, Storey \& Certo, 1999; Manetti et al., 2001; Shevlin \& O'Moore, 2000; Slininger et al., 2000), que propone que la técnica del contacto mejora las actitudes hacia las personas con discapacidad y el conocimiento sobre este tema, pero no consigue establecer vínculos más intensos (p. ej., afecto, amistad, cooperación, tolerancia). Además, para potenciar actitudes positivas se requiere promover la igualdad de todos los miembros del grupo y el fomento de actividades y tareas con metas y objetivos comunes (Pettigrew \& Trop, 2006; Wong, 2008). Puede ser que en los niños del GC, cuyo contacto se ha producido en un ambiente no estructurado, donde los niños con y sin discapacidad sí compartían espacios pero las actividades no se desarrollaban con una metodología cooperativa y no se aportaba información específica sobre temas relacionados con la discapacidad, el contacto va perdiendo eficacia y deja de ser una estrategia facilitadora de actitudes positivas, y de ahí que los niños de este GC desciendan sus puntuaciones en el último postest. No obstante, existe otro factor cuya relevancia en el cambio de tendencia no podemos obviar. La tesis que pone en relación la evolución de las actitudes y la edad mantiene que estas empeoran a medida que los niños se van haciendo mayores, especialmente a partir de la preadolescencia (Hugh, Guardia, Pires \& Rault, 2000; Nowicki, 2006; Pelechano, 1997). Incluso se ha llegado a encontrar correlación negativa entre edad y actitudes en niños de edades entre 8 y 12 años (Campbell, Ferguson, Herzinger, Jackson \& Marino, 2004). Este efecto de la edad no se aprecia en el GE en la última medida postest, momento en el que los niños ya tienen una edad de 12 años, es decir, ya son preadolescentes. Tal y como afirman Navas et al. (2004), es probable que la mejora de las actitudes con el paso del tiempo se produzca siempre y cuando vaya asociada a un mayor conocimiento de la discapacidad. Los chicos y chicas del GE, además del contacto, recibieron a lo 
largo del programa información variada y detallada sobre la discapacidad, conocimientos que se fueron revisando y actualizando a través de las actividades recordatorio a lo largo del período de seguimiento. En síntesis, se podría sostener que una metodología que recoja la introducción de actividades recordatorio posibilita un mejor mantenimiento del cambio de actitud conseguido, de tal forma que potencia la tendencia al alza de las puntuaciones del GE. Esta tendencia no la experimenta el GC en la última medida, cuando los niños también habían entrado en edad preadolescente.

Finalmente, es necesario hacer referencia a algunas de las limitaciones de este estudio. Por un lado, aquella derivada del hecho de haber trabajado con grupos naturales, lo que implica que los participantes no fuesen designados aleatoriamente al GE o al GC. Así, al desarrollar la investigación con grupos naturales, que se ofrecieron voluntariamente a participar en el programa, es previsible la existencia de variables extrañas que influyan en los resultados (p. ej., diferencias en la atención a la diversidad en los diferentes centros, o bien diferencias metodológicas de los docentes, entre otras muchas posibles variables). Por otro, sería conveniente realizar un seguimiento más prolongado en el tiempo para comprobar si lo ocurrido en los dos últimos postests, en los que se observaba cómo las puntuaciones del GE seguían subiendo mientras que las del GC bajaban de forma marcada, es un hecho puntual o es una tendencia. En coherencia, también se debe señalar que, para comprobar estadísticamente el efecto de las actividades recordatorio, se debería plantear un estudio en el que además del GC se contase con dos GE. Ambos GE participarían en el programa de cambio de actitudes, pero solo en uno de ellos se realizarían las actividades recordatorio. Un estudio con este diseño permitiría valorar con más exactitud la eficacia de las sesiones recordatorio.

Aún así, los resultados obtenidos permiten concluir que las actitudes se pueden cambiar, pero es preciso que ese cambio sea programado. A través de la información y contacto estructurado con personas con discapacidad se consigue que los niños mejoren sus actitudes hacia estas personas, y dicho cambio se mantiene en el tiempo, incluso al llegar a la preadolescencia, momento en el que suelen aparecer actitudes más negativas.

En definitiva, los resultados obtenidos sí justificarían la aplicación de un programa tan sencillo y económico como el que hemos realizado, lo que debería animar a la comunidad científica a seguir apostando por la implementación y posterior valoración de este tipo de estrategias tan estrechamente ligadas a la integración e inclusión educativa y social de los niños con discapacidad.

\section{Referencias}

Aguado, A. L., Alcedo, M. A. \& Arias, B. (2008). Cambio de actitudes hacia la discapacidad con escolares de primaria. Psicothema, 4(20), 697-704.

Aguado, A. L., Alcedo, M. A. \& Flórez, M. A. (2003). Un programa de cambio de actitudes hacia personas con discapacidad en entorno escolar. Análisis y Modificación de Conducta, 29(127), 673-704.

Aguado, A. L., Alcedo, M. A. \& Flórez, M. A. (2004). Programas de cambio de actitudes ante la discapacidad. Psicothema, 16(4), 667-673.

Almazán, L. (2003). Los cambios actitudinales hacia la integración escolar desde la perspectiva de los alumnos. Revista Fuentes, 5, 135-152.

Burns, M., Storey, K. \& Certo, N. J. (1999). Effect of service learning on attitudes towards students with severe disabilities. Education and Training in Mental Retardation and Developmental Disabilities, 34(1), 58-65.

Campbell, J. M., Ferguson, J. E., Herzinger, C. V., Jackson, J. N. \& Marino, C. A. (2004). Combined descriptive and explanatory information improves peers' perceptions of autism: A process of persuasive communication. Research in Developmental Disabilities, 25(4), 321-339.

Diamond, K. E., Hestenes, L., Carpenter, E. S. \& Innes, F. K. (1997). Relationships between enrollment in an inclusive class and preschool children's ideas about people with disabilities. Topics in Early Childhood Special Education, 17(4), 520-536.

España, Agencia Estatal Boletín Oficial del Estado. Ley Orgánica 4/2007, de 12 de abril, por la que se modifica la Ley Orgánica 6/2001, de 21 de diciembre, de Universidades. BOE, $\mathrm{n}$ ㅇ 89, de 13 de abril. 
España, Agencia Estatal Boletín Oficial del Estado. Real Decreto 1791/2010, de 30 de diciembre, por el que se aprueba el Estatuto del Estudiante Universitario. BOE, $n^{\circ}$ 318, de 31 de diciembre.

Fisher-Polites, C. (2004). "We all fit in": A program designed to promote understanding among typical children for children with disabilities. Journal of Positive Behavior Interventions, 6(3), 181-187.

Flórez, M. A., Aguado, A. L. \& Alcedo, M. A. (2009). Revisión y análisis de los programas de cambio de actitudes hacia personas con discapacidad. Anuario de Psicología Clínica y de la Salud, 5, 85-98.

García, L. G. A. \& Hernández, O. S. (2011). Actitudes hacia la discapacidad de jóvenes y adultos de Chiapas. Universitas Psychologica, 10(3), 817-827.

Hodge, S. R., Davis, R., Woodard, R. \& Sherrill, C. (2002). Comparison of practicum types in changing preservice teachers' attitudes and perceived competence. Adapted Physical Activity Quarterly, 19(2), 155-171.

Huete, A., Sola, A. \& Lara, P. (2010). Los jóvenes con discapacidad en España. Informe de situación 2010. Madrid: CERMI.

Hugh, S., Guardia, M., Pires, M. \& Rault, C. (2000). Attitudes towards Down syndrome: A national comparative study: France, Ireland, Portugal and Spain. Irish Journal of Psychology, 21(3-4), 203-214.

Instituto Asturiano de Estadística. (2011). Datos básicos de Asturias 2011. Oviedo: Gobierno del Principado de Asturias.

Krahé, B. \& Altwasser, C. (2006). Changing negative attitudes towards persons with physical disabilities: An experimental intervention. Journal of Community Ë Applied Social Psychology, 16(1), 59-69.

Laws, G. \& Kelly, E. (2005). The attitudes and friendship intentions of children in United Kingdom Mainstream Schools towards peers with physical or intellectual disabilities. International Journal of Disability, Development and Education, 52(2), 79-99.

Magiati, I., Dockrell, J. \& Longotheti, A. (2002). Young children's understanding of disabilities: The influence of development, context and cognition. Applied Developmental Psychology, 23(4), 409-430.

Manetti, M., Schenider, B. H. \& Siperstein, G. (2001). Social acceptance of children with mental retardation: Testing the contact hypothesis with an
Italian sample. International Journal of Behavioural Development, 23(3), 279-286.

Maras, P. \& Brown, R. (2000). Effects of different forms of school contact on children's attitudes toward disabled and non-disabled peers. British Journal of Educational Psychology, 70(3), 337-351.

McCarthy, J. \& Light, J. (2005). Attitudes toward individuals who use augmentative and alternative communication: Research review. Augmentative and Alternative Communication, 21(1), 41-55.

McDougall, J., DeWit, D. J., King, G., Miller, L. T. \& Killip, S. (2004). High school-aged youth's attitudes toward their peers with disabilities: The role of school and student interpersonal factors. International Journal of Disability, Development and Education, 51(3), 287-313.

Morton, J. F. \& Campbell, J. M. (2007). Information source affects peers' initial attitudes toward autism. Research in Developmental Disabilities, 29(3), 189-201.

Navas, L., Torregrosa, G. \& Mula, A. (2004). Algunas variables predictoras de las actitudes del alumnado ante la integración escolar. Revista de Psicología Social, 19(2), 159-171.

Nikolaraizi, M. \& De Reybekiel, N. (2001). A comparative study of children's attitudes towards deaf children, children in wheelchairs and blind children in Greece and in the UK. European Journal of Special Needs Education, 16(21), 167-182.

Nowicki, E. A. (2006). A cross-sectional multivariate analysis of children's attitudes towards disabilities. Journal of Intellectual Disability Research, 50(5), 335-348.

Nowicki, E. A. \& Sandieson, R. (2002). A meta-analysis of school-age children's attitudes towards persons with physical or intellectual disabilities. International Journal of Disability, Development and Education, 49(3), 243-265.

Organización Mundial de la Salud \& Grupo del Banco Mundial. (2011). Informe mundial sobre la discapacidad. Ginebra: WHO. Disponible en http://whqlibdoc.who.int/hq/2011/WHO_NMH_VIP_11.03_ spa.pdf

Pelechano, V. (1997, marzo). Determinantes de las actitudes ante la integración y su modificación. Conferencia presentada en II Jornadas Científicas de 
Investigación sobre personas con discapacidad, Salamanca, España.

Peralta, A. (2007). Libro blanco sobre universidad y discapacidad. Madrid: Real Patronato sobre Discapacidad, CERMI/Fundación Vodafone España/ ANECA.

Pettigrew, T. F. \& Trop, L. R. (2006). A meta-analytic test of intergroup contact theory. Journal of Personality and Social Psychology, 90(5), 751-783.

Polo, M. T., Fernández, C. \& Díaz, C. (2011). Estudio de las actitudes de estudiantes de Ciencias Sociales y Pedagogía. Universitas Psychologica, 10(1), 113-123.

Rillota F. \& Nettelbeck, T. (2007). Effects of an awareness program on attitudes of students without an intellectual disability towards persons with an intellectual disability. Journal of Intellectual $\mathbb{E}$ Developmental Disability, 32(1), 19-27.
Shevlin, M. \& O'Moore, A. M. (2000). Fostering positive attitudes: Reactions of mainstream pupils to contact with their counterparts who have severe/ profound intellectual disabilities. European Journal of Special Needs Education, 15(2), 206-217.

Slininger, D., Sherrill, C. \& Jankowski, C. M. (2000). Children's attitudes toward peers with severe disabilities: Revisiting contact theory. Adapted Physical Activity Quarterly, 17(2), 176-196.

Verdugo, M. A., Arias, B. \& Jenaro, C. (1994). Actitudes hacia las personas con minusvalía. Madrid: Ministerio de Asuntos Sociales, INSERSO.

Wong, D. (2008). Do contacts make a difference? The effects of mainstreaming on student attitudes toward people with disabilities. Research in Developmental Disabilities, 29(1), 70-82. 